\title{
Genome-wide identification of MAPKKK genes and their responses to phytoplasma infection in Chinese jujube (Ziziphus jujuba Mill.)
}

Zhiguo Liu ${ }^{1,2+}$, Lixin Wang ${ }^{1,2+}$, Chaoling Xue ${ }^{3,4}$, Yuetong Chu ${ }^{1,2}$, Weilin Gao ${ }^{3,4}$, Yitong Zhao ${ }^{3,4}$, Jin Zhao Zh $^{3,4^{*}}$ and Mengjun Liu ${ }^{1,2^{*}}$

\begin{abstract}
Background: Mitogen-activated protein kinase (MAPK) cascades play vital roles in signal transduction in response to a wide range of biotic and abiotic stresses. In a previous study, we identified ten ZjMAPKs and five ZjMAPKKs in the Chinese jujube genome. We found that some members of ZjMAPKs and ZjMAPKKs may play key roles in the plant's response to phytoplasma infection. However, how these ZjMAPKKs are modulated by ZjMAPKKKKs during the response process has not been elucidated. Little information is available regarding MAPKKKs in Chinese jujube.

Results: A total of 56 ZjMAPKKKs were identified in the jujube genome. All of these kinases contain the key S-TKc (serine/threonine protein kinase) domain, which is distributed among all 12 chromosomes. Phylogenetic analyses show that these ZjMAPKKKs can be classified into two subfamilies. Specifically, 41 ZjMAPKKKs belong to the Raf subfamily, and 15 belong to the MEKK subfamily. In addition, the ZjMAPKKKS in each subfamily share the same conserved motifs and gene structures. Only one pair of ZjMAPKKKs (15/16, on chromosome 5) was found to be tandemly duplicated. Using qPCR, the expression profiles of these MAPKKKs were investigated in response to infection with phytoplasma. In the three main infected tissues (witches' broom leaves, phyllody leaves, and apparently normal leaves), ZjMAPKKK26 and - 45 were significantly upregulated, and ZjMAPKKK3, -43 and - 50 were significantly downregulated. ZjMAPKKK4, $-10,-25$ and -44 were significantly and highly induced in sterile cultivated tissues infected by phytoplasma, while ZjMAPKKK6, $-7,-17,-18,-30,-34,-35,-37,-40,-41,-43$, $-46,-52$ and -53 were significantly downregulated.
\end{abstract}

Conclusions: For the first time, we present an identification and classification analysis of ZjMAPKKKs. Some ZjMAPKKK genes may play key roles in the response to phytoplasma infection. This study provides an initial understanding of the mechanisms through which ZjMAPKKKs are involved in the response of Chinese jujube to phytoplasma infection.

Keywords: Chinese jujube, MAPKKKs, Jujube witches' broom, Phytoplasma, Expression profiles

\footnotetext{
* Correspondence: zhaojinbd@126.com; Imj1234567@aliyun.com

${ }^{\dagger}$ Zhiguo Liu and Lixin Wang contributed equally to this work.

${ }^{3}$ College of Life Science, Hebei Agricultural University, Baoding, China

${ }^{1}$ College of Horticulture, Hebei Agricultural University, Baoding, China

Full list of author information is available at the end of the article
}

(c) The Author(s). 2020 Open Access This article is distributed under the terms of the Creative Commons Attribution 4.0 International License (http://creativecommons.org/licenses/by/4.0/), which permits unrestricted use, distribution, and reproduction in any medium, provided you give appropriate credit to the original author(s) and the source, provide a link to the Creative Commons license, and indicate if changes were made. The Creative Commons Public Domain Dedication waiver (http://creativecommons.org/publicdomain/zero/1.0/) applies to the data made available in this article, unless otherwise stated. 


\section{Background}

Mitogen activated protein kinase (MAPK) cascades comprise three specific kinase families: MAP kinase kinase kinases (MAPKKKs), MAP kinase kinases (MAPKKs) and MAP kinases (MAPKs). Essentially, these kinase families are intermediate signalling modules that operate between signal sensing and the activation of related transcription factors. These kinases are involved in plant responses to biotic and abiotic stresses, such as drought, salinity, cold and pathogen attack [1-3]. The conserved serine/threonine MAPKKKs can be activated by plasma membrane receptors which, in turn, phosphorylate MAPKKs, which then activate MAPKs by sequential phosphorylation. Finally, MAPKs regulate other kinases or related transcription factors in response to various stresses [1, 4]. Each MAPK cascade family consists of a number of members, and the number of members varies significantly between families. For example, the MAPKKK family comprises a greater number of members and shows more complex sequence diversity than the other two families. The members belonging to the MAPKKK family can be classified into the subfamilies Raf, ZIK and MEKK according to their characteristic sequence motifs [5]. Structural diversity is found among MAPKKKs in each subfamily. The Raf subfamily has a C-terminal kinase domain and a long N-terminal regulatory domain, while ZIK proteins have only N-terminal kinase domains, and the MEKK subfamily has fewer conserved kinase domains. In addition, a long Nterminal regulatory domain forms the backbone for the Raf and ZIK subfamilies [1, 5].

MAPK cascades have been implicated in signal transduction in distinct innate immunity [6, 7]. In Arabidopsis, the MEKK1-MKK4/5-MPK3/6-WRKY22/WRKY29/ FRK1 cascade is involved in innate immunity signal transduction, and the MEKK1-MKK1/MKK2-MPK4 kinase cascade can negatively activate $M E K K 2$, which further leads to SUMM2-mediated immune responses [8, 9]. In tobacco, NPK1-MEK1-Ntf6 can regulate WRKY/ $M Y B$ transcription factors to participate in the tobacco mosaic virus infection pathway [10]. In addition, the $M A P K K K \alpha-M K K 2 / M K K 4-M P K 2 / M P K 3$ cascades participate in the Pto-mediated effect or triggered immunity (ETI) pathway by regulating the transcription factor TGA in tomato [11]. Hence, the MAPK pathway is indeed involved in the response to pathogen attack and may also play essential roles in the response to phytoplasma infection in Chinese jujube.

The $M A P K K K$ family has now been characterised in the plant kingdom. A total of $80 M A P K K K S$ were first identified in Arabidopsis in 2002 [5, 12]. In the following years, an array of different MAPKKKS have been identified in a range of plant species, including rice $(75$ members), Zea mays (71 members), Vitis vinifera (45 members), Malus domestica (72 members) and Musa nana (77 members) [13-16]. However, little is known about the biological information and function of the $M A P K K K$ gene family in Chinese jujube, even though detailed information for ZjMAPKKs and ZjMAPKs has previously been reported by our group [17].

Jujube witches' broom disease (JWB) is caused by 'Candidatus Phytoplasma ziziphi'. JWB is a devastating disease in Asia [18]. Since the 1990s in China and with no effective control methods, JWB disease has severely impacted yields of Chinese jujube [19]. Our group has focused on this disease for many years, and we have published a book, 'Jujube Witches' Broom Disease', which provides detailed information on a number of key questions, including how the phytoplasma infects the plant with a one-year life cycle, how to test for JWB and how to evaluate the severity of JWB. Typical symptoms that can be observed in a plant suffering phytoplasma disease include witches' broom and phyllody. The physiological and biochemical behaviours of jujube plants infected by this phytoplasma have been widely studied [19-21], but the underlying molecular mechanisms have not been elucidated. Recently, MAPKs have been reported in response to phytoplasma infection of Chinese jujube with analysis of their expression levels in different phytoplasma-infected materials [22]. This study has provided valuable insights into the important roles played by MAPKs during the infection process. In addition, ZjMPK2, ZjMKK2 and ZjMKK4 have been shown to be the main genes involved in the Chinese jujube-phytoplasma interaction. Additionally, using yeast two-hybrid analyses, it has been demonstrated that ZjMKK2 interacts with ZjMPK2 [17, 23]. All these results demonstrate the important function of MAPK cascades in response to phytoplasma infection in Chinese jujube. However, identification and initial functional analyses of ZjMAPKKKS are needed to build our knowledge of the complete MAPK cascade signalling transduction pathway. Thus, the aim of this study was to identify ZjMAPKKKS using genome-wide and phylogenetic analyses to predict the gene structure and conserved motifs of ZjMAPKKKs. Then, the expression profiles of these ZjMAPKKKS in response to phytoplasma infection were investigated by qPCR. The end goal is to expand our understanding of the mechanism through which ZjMAPKKKS are involved in the defence responses of Chinese jujube to witches' broom disease.

\section{Results}

\section{Genome-wide identification of ZjMAPKKKs}

A total of 56 ZjMAPKKKs were defined. All of these kinases have the key S-TKc (serine/threonine protein kinase) domain and other conserved protein kinase domains (Additional file 2 Table S1). To clearly understand and discriminate between the MAPKKK genes, the locus of $Z j M A P K K K s$ was designated according to the nomenclature 
suggestions for Arabidopsis, where $Z j$ refers to Ziziphus jujuba, and the series numbers ZjMAPKKK1-56 are coded in terms of their chromosome locations (Table 1). The $Z j M A P K K K s$ are distributed over all 12 pseudochromosomes, except for ZjMAPKKK44-56, which could not be matched to a corresponding chromosome.

Specific information for each CDS and amino acid sequence of the ZjMAPKKKS is listed in Additional files 1 and 7. Based on the specific conserved signature motif, all the ZjMAPKKKS could be grouped into one of the two subfamilies Raf and MEKK. No ZIK subfamily members were identified. As shown in Table 1, the length of the CDS sequences ranged from $762 \mathrm{bp}$ (ZjMAPKKK36) to $4455 \mathrm{bp}$ (ZjMAPKKK7) with an average length of $1804 \mathrm{bp}$. The amino acid sequence length of ZjMAPKKKs varied from 253 (ZjMAPKKK36) to 1484 (ZjMAPKKK7) amino acids (aa); the average length was 600 aa. The predicted molecular weight $(\mathrm{Mw})$ of these proteins ranged from 28.29 (ZjMAPKKK36) to 160.66 (ZjMAPKKK7), and the theoretical isoelectric points $(\mathrm{pI})$ ranged from 4.78 to 9.34 .

\section{Phylogenetic analyses of ZjMAPKKK genes}

To assess the phylogenetic relationships between Chinese jujube and Arobidopsis, a phylogenetic tree was constructed with all 136 protein sequences (56 ZjMAPKKKs and 80 AtMAPKKKs). As illustrated in Fig. 1, the members of AtMAPKKKs could be clustered into three categories, Raf, ZIK and MEKK, indicating that the method used to build the phylogenetic tree was reliable. However, the 56 members of ZjMAPKKKs could be clustered into only two subfamilies, Raf and MEKK. In addition, the largest Raf subfamily consisted of 41 members, with the remaining 15 members of ZjMAPKKKs belonging to the MEKK subfamily. None could be ascribed to the ZIK subfamily. Moreover, some ZjMAPKKKs located on the same chromosome showed little divergence but clustered into the same group. Examples are ZjMAPKKK36, - 37 and 40; ZjMAPKKK15 and - 16; and ZjMAPKKK38 and - 39. These results indicate that some duplication of ZjMAPKKKs occurred during the evolution of jujube.

\section{Conserved domains and gene structure analyses of ZjMAPKKKs}

Within the analysis of MEME software, five main conserved motifs were identified in all 56 ZjMAPKKKs (Fig. 2). Motifs 1, 3 and 4 were found in all ZjMAPKKKs, while the other two motifs were observed in all Raf subfamily members. The MEKK subfamily members fell into two groups: one contained motifs 1-4, including ZjMAPKKK21, - 56, - 6, $-31,-10$ and -25 . The remaining members contained only motifs 1,3 and 4 . These results illustrate that ZjMAPKKKs share the same conserved motifs, which further indicates that the protein structures for each subfamily are highly conserved.
For the analyses of the exon/intron contents, the differences among ZjMAPKKKs were significant. As shown in Fig. 3 and in Additional file 8Table S3, the number of exons in ZjMAPKKKs ranged from 1 (ZjMAPKKK9, - 12, - 29, 35, - 36, - 44 and - 54) to 19 (ZjMAPKKK42). Interestingly, the members of ZjMAPKKKs containing only 1 exon all belong to the MEKK subfamily (47\%). The highest number of exons in this subfamily was 17 (ZjMAPKKK25 and -10), and the average number was 5.6. This result demonstrates that in this subfamily, significant loss and gains of exons took place during evolution. For the Raf subfamily, the number of exons varied from 2 (ZjMAPKKK16 and -28) to 19 (ZjMAPKKK42) with an average number of 9.56. Although there was significant variation in the number of exons in the Raf and MEKK subfamilies, some exon structure patterns were clearly conserved in close paralogues. For instance, ZjMAPKKK24 and -49 have 12 exons, while ZjMAPKKK37 and -40 have 2 exons, and they are all closely clustered in the same phylogenetic tree. Collectively, the evolutionarily different organisations of the ZjMAPKKK gene structures between the Raf and MEKK subfamilies indicate that tandem and segmental duplication events may have occurred in ancient times and that diverse exon structures may function differently in the jujube genome.

Furthermore, with the multiple protein alignment of ZjMAPKKKs, the Raf-specific signature motif GTXX(W/ Y) MAPE was found in the Raf subfamily, and the kinase domain was located at the $\mathrm{N}$ terminal or $\mathrm{C}$ terminal. In contrast, the less conserved MEKK-specific signature motif $G(T / S) P X(W / F)$ MAPEV was observed in the MEKK subfamily, while the kinase domain was located at three positions: the $\mathrm{N}$ - or C-terminal or the central part of the proteins (Fig. 4). The features of the signature motifs of ZjMAPKKKs are consistent with other orthologues in other plant species, where they fulfil important roles in a diversity of signal transduction processes.

\section{Synteny analysis of ZjMAPKKK genes}

Tandem duplication events were first analysed according to the principle that two or more genes can be located on a chromosomal region within $200 \mathrm{~kb}$ [24] of one another. As shown in Fig. 5, one pair of ZjMAPKKKs (15/16) was the only tandem duplication event on LG5. In addition, 13 segmental duplication events with 22 ZjMAPKKKs were also identified. These results indicate that some $Z j M A P K K K s$ may have been generated by gene duplication and that segmental duplication events were probably a major driving force in ZjMAPKKK evolution.

\section{Phytoplasma detection in different tissues infected by phytoplasma}

To characterize the functions of ZjMAPKKKs involved in phytoplasma infection, the expression levels of individual ZjMAPKKKs were detected by qPCR in two kinds of 
Table 1 Characteristic of MAPK Kinase Kinases from Ziziphus jujuba Mill. (ZjMAPKKKs)

\begin{tabular}{|c|c|c|c|c|c|c|c|c|}
\hline Group & Name & Locus ID & Chr & Location & CDS (bp) & Amino acid length (AA) & $\mathrm{PI}$ & $\mathrm{MW}(\mathrm{KD})$ \\
\hline & ZjMAPKKK1 & LOC107412947 & Chr1 & $512,097-518,184$ & 3351 & 1116 & 5.56 & 123.87 \\
\hline & ZjMAPKKK2 & LOC101223021 & Chr1 & $588,730.592155$ & 1062 & 353 & 7.16 & 39.83 \\
\hline & ZjMAPKKK3 & LOC107413171 & Chr1 & $6,034,745.6041047$ & 1380 & 459 & 8.99 & 52.10 \\
\hline & ZjMAPKKK4 & LOC107414729 & Chr1 & 7,211,380..7216386 & 1119 & 372 & 9.01 & 42.35 \\
\hline & ZjMAPKKK5 & LOC107422643 & Chr1 & $17,322,629.17330021$ & 1707 & 568 & 6.55 & 64.31 \\
\hline & ZjMAPKKK7 & LOC107427772 & Chr1 & $28,684,428.28693993$ & 4455 & 1484 & 5.27 & 160.66 \\
\hline & ZjMAPKKKR & LOC107429777 & Chr1 & $31,236,415.31242279$ & 3432 & 1143 & 7.67 & 126.67 \\
\hline & ZjMAPKKK11 & LOC107412267 & Chr2 & $23,790,620.23798118$ & 2913 & 970 & 5.83 & 107.05 \\
\hline & ZjMAPKKK13 & LOC107415263 & Chr4 & $1,873,602 . .1877368$ & 1059 & 352 & 7.66 & 39.46 \\
\hline \multirow[t]{18}{*}{ Raf } & ZjMAPKKK14 & LOC107417160 & Chr4 & $23,620,629.23633413$ & 2208 & 735 & 6.1 & 83.02 \\
\hline & ZjMAPKKK15 & LOC107417666 & Chr5 & $2,301,847 . .2306994$ & 1248 & 415 & 8.14 & 46.31 \\
\hline & ZjMAPKKK16 & LOC107417677 & Chr5 & $2,314,642.2318435$ & 1257 & 418 & 7.62 & 46.80 \\
\hline & ZjMAPKKK17 & LOC107417907 & Chr5 & $5,814,146.5820584$ & 3789 & 1262 & 5.18 & 139.68 \\
\hline & ZjMAPKKK18 & LOC107417903 & Chr5 & $5,822,068.5828809$ & 3825 & 1274 & 5.43 & 140.42 \\
\hline & ZjMAPKKK19 & LOC107418405 & Chr5 & $10,268,020.10272429$ & 1074 & 357 & 8.97 & 40.45 \\
\hline & ZjMAPKKK20 & LOC107418996 & Chr5 & $18,139,031 . .18145136$ & 2343 & 780 & 6.38 & 87.04 \\
\hline & ZjMAPKKK22 & LOC107421353 & Chr6 & $17,987,031 . .17997512$ & 2832 & 943 & 8.15 & 105.39 \\
\hline & ZjMAPKKK23 & LOC107420099 & Chr6 & $2,889,952.2898479$ & 2856 & 951 & 5.53 & 104.94 \\
\hline & ZjMAPKKK24 & LOC107422567 & Chr7 & $14,102,760.14109942$ & 3444 & 1147 & 5.87 & 127.99 \\
\hline & ZjMAPKKK26 & LOC107423093 & Chr7 & $21,216,345.21219607$ & 1173 & 390 & 7.92 & 43.53 \\
\hline & ZjMAPKKK27 & LOC107423594 & Chr7 & $27,636,786.27641226$ & 1251 & 416 & 6.11 & 46.81 \\
\hline & ZjMAPKKK28 & LOC107424157 & Chr8 & $4,344,698 . .4348053$ & 2049 & 682 & 8.81 & 79.10 \\
\hline & ZjMAPKKK30 & LOC107424832 & Chr8 & $9,154,812 . .9160628$ & 1179 & 392 & 9.11 & 44.23 \\
\hline & ZjMAPKKK32 & LOC107426395 & Chr9 & $4,943,575 . .4946720$ & 1203 & 400 & 6.29 & 44.66 \\
\hline & ZjMAPKKK33 & LOC107426719 & Chr9 & $5,733,599.5742140$ & 3945 & 1314 & 5.32 & 144.52 \\
\hline & ZjMAPKKK34 & LOC107427400 & Chr9 & $18,534,579 . .18538786$ & 1032 & 343 & 5.84 & 38.71 \\
\hline & ZjMAPKKK38 & LOC107428906 & Chr10 & $10,418,263 . .10423580$ & 1299 & 432 & 8.15 & 48.84 \\
\hline \multirow[t]{14}{*}{ Raf } & ZjMAPKKK39 & LOC107428931 & Chr10 & $11,520,310 . .11525561$ & 1299 & 432 & 7.74 & 48.89 \\
\hline & ZjMAPKKK41 & LOC107430036 & Chr11 & 2,650,983..2657196 & 2181 & 726 & 6.97 & 81.01 \\
\hline & ZjMAPKKK42 & LOC107431473 & Chr11 & $19,821,853.19829329$ & 1707 & 568 & 5.51 & 64.02 \\
\hline & ZjMAPKKK43 & LOC107432147 & Chr12 & $5,159,272.5168476$ & 2556 & 851 & 5.98 & 93.67 \\
\hline & ZjMAPKKK45 & LOC107408109 & Unplaced Scaffold & 688.6689 & 1425 & 474 & 9.12 & 53.23 \\
\hline & ZjMAPKKK46 & LOC107405634 & Unplaced Scaffold & $5820 . .12905$ & 1341 & 446 & 5.58 & 50.42 \\
\hline & ZjMAPKKK47 & LOC107407393 & Unplaced Scaffold & $6471 . .11148$ & 1125 & 374 & 7.13 & 42.29 \\
\hline & ZjMAPKKK48 & LOC107406964 & Unplaced Scaffold & $13,195 . .15977$ & 1005 & 334 & 7.68 & 37.91 \\
\hline & ZjMAPKKK49 & LOC107404883 & Unplaced Scaffold & $14,404 . .17983$ & 1251 & 416 & 6.24 & 46.77 \\
\hline & ZjMAPKKK50 & LOC107406505 & Unplaced Scaffold & $16,102 . .20361$ & 1059 & 352 & 7.17 & 39.84 \\
\hline & ZjMAPKKK51 & LOC107405705 & Unplaced Scaffold & $33,936 . .39585$ & 1158 & 385 & 7.51 & 42.93 \\
\hline & ZjMAPKKK52 & LOC107403422 & Unplaced Scaffold & $48,143 . .64604$ & 1647 & 548 & 5.13 & 61.65 \\
\hline & ZjMAPKKK53 & LOC107435406 & Unplaced Scaffold & $61,008.63977$ & 1479 & 492 & 9.34 & 56.56 \\
\hline & ZjMAPKKK55 & LOC107435407 & Unplaced Scaffold & $134,848 . .138183$ & 1482 & 493 & 9.22 & 56.35 \\
\hline \multirow[t]{3}{*}{ MEKK } & ZjMAPKKK6 & LOC107423632 & Chr1 & $18,688,160.18695042$ & 2700 & 899 & 9.29 & 96.87 \\
\hline & ZjMAPKKK9 & LOC107432528 & Chr1 & $34,451,996.34453515$ & 1428 & 475 & 4.78 & 53.09 \\
\hline & ZjMAPKKK10 & LOC107411974 & Chr2 & $21,809,712.21815908$ & 2046 & 681 & 5.64 & 74.86 \\
\hline
\end{tabular}


Table 1 Characteristic of MAPK Kinase Kinases from Ziziphus jujuba Mill. (ZjMAPKKKs) (Continued)

\begin{tabular}{|c|c|c|c|c|c|c|c|c|}
\hline Group & Name & Locus ID & Chr & Location & CDS (bp) & Amino acid length (AA) & $\mathrm{PI}$ & $\mathrm{MW}(\mathrm{KD})$ \\
\hline & ZjMAPKKK12 & LOC107414154 & Chr3 & $19,912,625 . .19913971$ & 1266 & 421 & 4.95 & 46.85 \\
\hline & ZjMAPKKK21 & LOC107420999 & Chr6 & $10,913,503 . .10919747$ & 1839 & 612 & 5.53 & 67.87 \\
\hline & ZjMAPKKK25 & LOC107423026 & Chr7 & $20,770,505 . .20775489$ & 2058 & 685 & 6.78 & 75.80 \\
\hline & ZjMAPKKK29 & LOC107424505 & Chr8 & $6,765,530 . .6767083$ & 1071 & 356 & 4.93 & 39.97 \\
\hline & ZjMAPKKK31 & LOC107425633 & Chr8 & $20,944,047 . .20949800$ & 1563 & 520 & 9.11 & 56.65 \\
\hline & ZjMAPKKK35 & LOC107427543 & Chr9 & $20,198,543 . .20199481$ & 939 & 312 & 6.52 & 35.52 \\
\hline & ZjMAPKKK36 & LOC107428154 & Chr10 & $1,469,136 . .1470695$ & 762 & 253 & 7.01 & 28.29 \\
\hline & ZjMAPKKK37 & LOC107428813 & Chr10 & $8,441,149 . .8442948$ & 825 & 274 & 4.89 & 30.43 \\
\hline & ZjMAPKKK40 & LOC107429056 & Chr10 & $13,522,021 . .13523553$ & 1035 & 344 & 5.36 & 37.67 \\
\hline & ZjMAPKKK44 & LOC107409320 & Unplaced Scaffold & $335 . .2009$ & 1335 & 444 & 4.8 & 50.18 \\
\hline & ZjMAPKKK54 & LOC107434197 & Unplaced Scaffold & $108,777 . .110183$ & 1119 & 372 & 5.46 & 41.39 \\
\hline & ZjMAPKKK56 & LOC107435014 & Unplaced Scaffold & $155,489.160603$ & 1836 & 611 & 5.64 & 67.84 \\
\hline
\end{tabular}

Note: Chr: chromosome; Pl: the theoretical isoelectric point of proteins; MW: The theoretical molecular weight of proteins

infected plant material. The first infected plant material was from diseased plants in the field (in vivo). This material showed three levels of symptoms: (a) witches' broom leaves, (b) phyllody leaves and (c) apparently normal leaves (but from diseased plants). The other infected plant material was from sterile (in vitro) cultured tissues of JWB plantlets. The phytoplasma concentrations in the in vivo material with three levels of symptoms were measured by Xue et al. (2018) [21]. The phytoplasma determination in the in vitro tissues shows fluorescent spots forming a large circle in the phloem of the petiole (Additional file 5Figure S4). These results confirm the subsequent tests on ZjMAPKKK function in response to phytoplasma infection.

\section{Expression analysis of ZjMAPKKKs in witches' broom leaves}

In Additional file 9 Table S4 and Fig. 6(a), the heat map shows the expression levels of ZjMAPKKKs with significantly different patterns in the witches' broom leaves from June to September. There were 42 candidates expressing at a detectable level, but the expression levels of the other 14 $Z j M A P K K K s$ were either not expressed or were expressed at levels below our detection threshold. The ZjMAPKKKS genes with too low (or nonexistent) expression were rejected as candidates for further calculation and analysis. Among these genes, the most significant transcript induction took place in the early stage (June or July) when the concentration of witches' broom began to increase. For example, ZjMAPKKK13, - 14, - 15, - 23, - 34, - 42, - 44, 47 and - 56 were significantly induced in June or July, but induction later decreased from August to September, as shown in Fig. 6(b). However, ZjMAPKKK3, - 43 and - 50 were downregulated from June to September. The expression levels of two ZjMAPKKK members (ZjMAPKKK26 and -45$)$ remained high from June to September. However, the clustering of ZjMAPKKK expression profiles was not aligned with gene similarities, illustrating that gene function may not rely on gene structure.

\section{Expression analysis of ZjMAPKKKs in phyllody leaves}

The transcript abundance of ZjMAPKKKs was also investigated in phyllody leaves. The heat map of the expressing ZjMAPKKKs is shown in Fig. 7(a). Several of the ZjMAPKKKS were highly expressed in June or July, but their expression levels then decreased from August to September. However, most ZjMAPKKKs showed no significant changes in expression level. Expression details for all ZjMAPKKKS are shown in Fig. 7(b). ZjMAPKKK10, - 14, - $15-34,-44$ and - 56 were all significantly upregulated in the early stage (June or July). However, 10 of the ZjMAPKKKs (ZjMAPKKK3, - 16, $18,-41,-43,-50,-51,-52,-53$ and -55$)$ were significantly downregulated. As in the witches' broom leaves, in the phyllody leaves, ZjMAPKKK26 and -45 were highly upregulated from June to September.

\section{Expression analysis of ZjMAPKKKs in apparently normal leaves}

The apparently normal but asymptomatic infected leaves were used to test which $Z j M A P K K K S$ play a role in the phytoplasma infection response. Interestingly, the heat map figure shows different expression patterns for $Z j M A P K K K S$ in these leaves (Fig. 8b). A few genes were highly upregulated, but most showed downregulation. For example, ZjMAPKKK1, - 3, - 7, - 16, - 17, - 18, $19,-41,-43,-50$, and -52 were downregulated from June to September, while ZjMAPKKK28, - 34 and - 47 were significantly upregulated in June or July, while ZjMAPKKK27 and - 54 were upregulated from August or September. However, ZjMAPKKK26 and - 45 showed the same pattern of high expression in the asymptomatic infected leaves from June to September (Fig. 8a). 


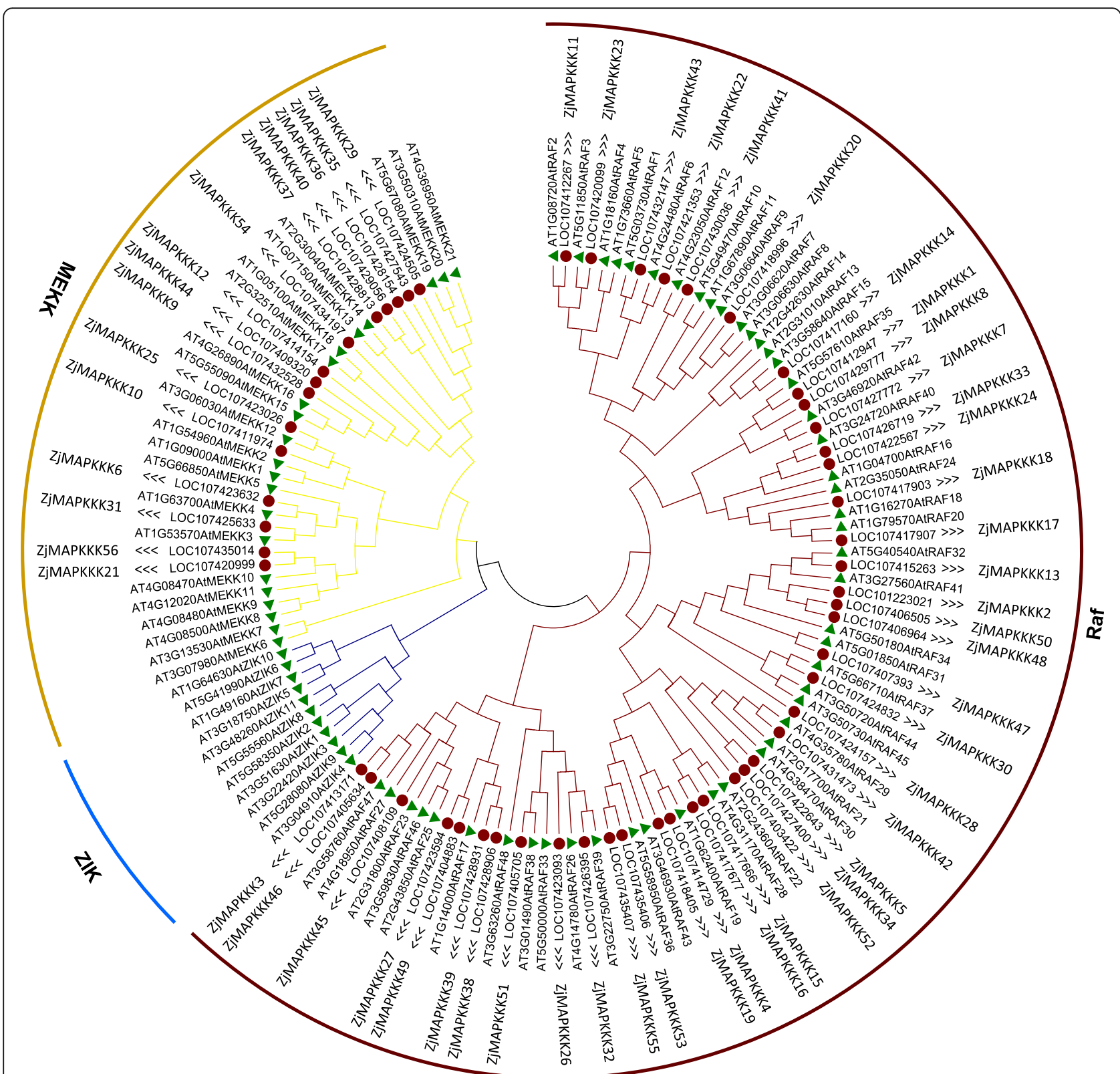

Fig. 1 Phylogenetic analyses of ZjMAPKKKs (Ziziphus jujuba Mill.) and AtMAPKKKs (Arabidopsis thaliana) with a total of 136 protein sequences. MEGA 6.0 was used to construct the phylogenetic tree employing the neighbour-joining ( $\mathrm{NJ}$ ) method. A total of 1000 bootstrap replications were carried out to indicate reliability. The ZjMAPKKKs were clustered into two groups - the Raf and MEKK subfamilies

To summarise, in the phytoplasma-infected tissues of the three symptomatic severities (apparently normal, phyllody and witches' broom) and in the 4 months (June through September), ZjMAPKKK26 was significantly upregulated, and ZjMAPKKK 45 was also highly induced. As the infection developed, the visible disease symptoms increased, becoming gradually more severe, from apparently normal leaves to phyllody leaves to witches' broom leaves [20]. This progression occurred even though the concentration of JWB decreased gradually from August through September.
The expression of ZjMAPKKK26 increased approximately six-fold in the phyllody leaves in June but not at the other two symptomatic levels. Then, as the infection developed, ZjMAPKKK26 was upregulated approximately three-fold in the witches' broom leaves in July but downregulated in the phyllody. Meanwhile, in June, the induction of ZjMAPKKK45 increased approximately three-fold in the apparently normal (but phytoplasma-infected) leaves and approximately sixfold in the phyllody leaves. Then, during July, August and September, it was downregulated, but induction 

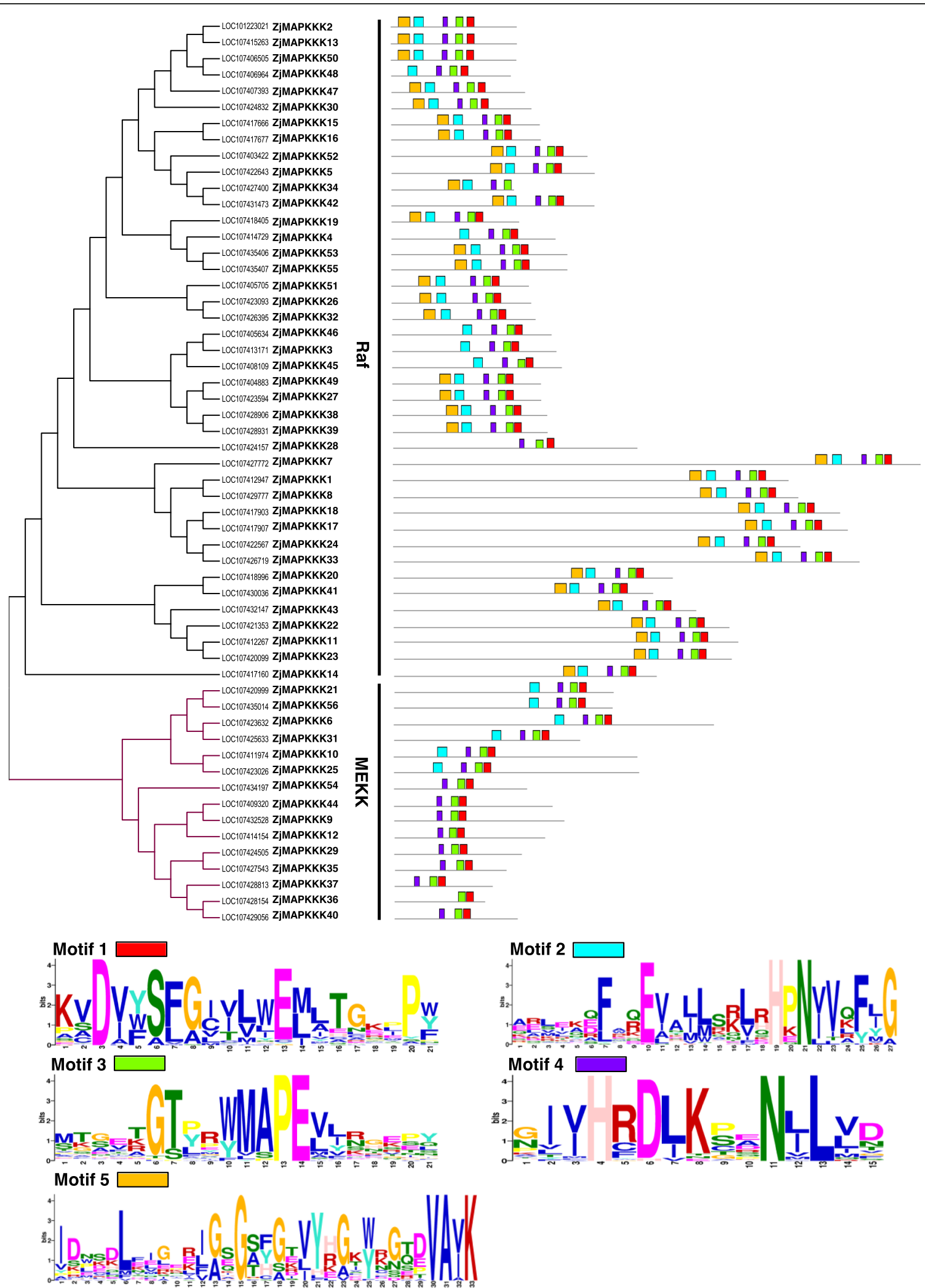

Fig. 2 Identification of the conserved motifs of ZjMAPKKKs corresponding to the phylogenetic tree. The MEME database was used to identify the motifs based on protein sequences 


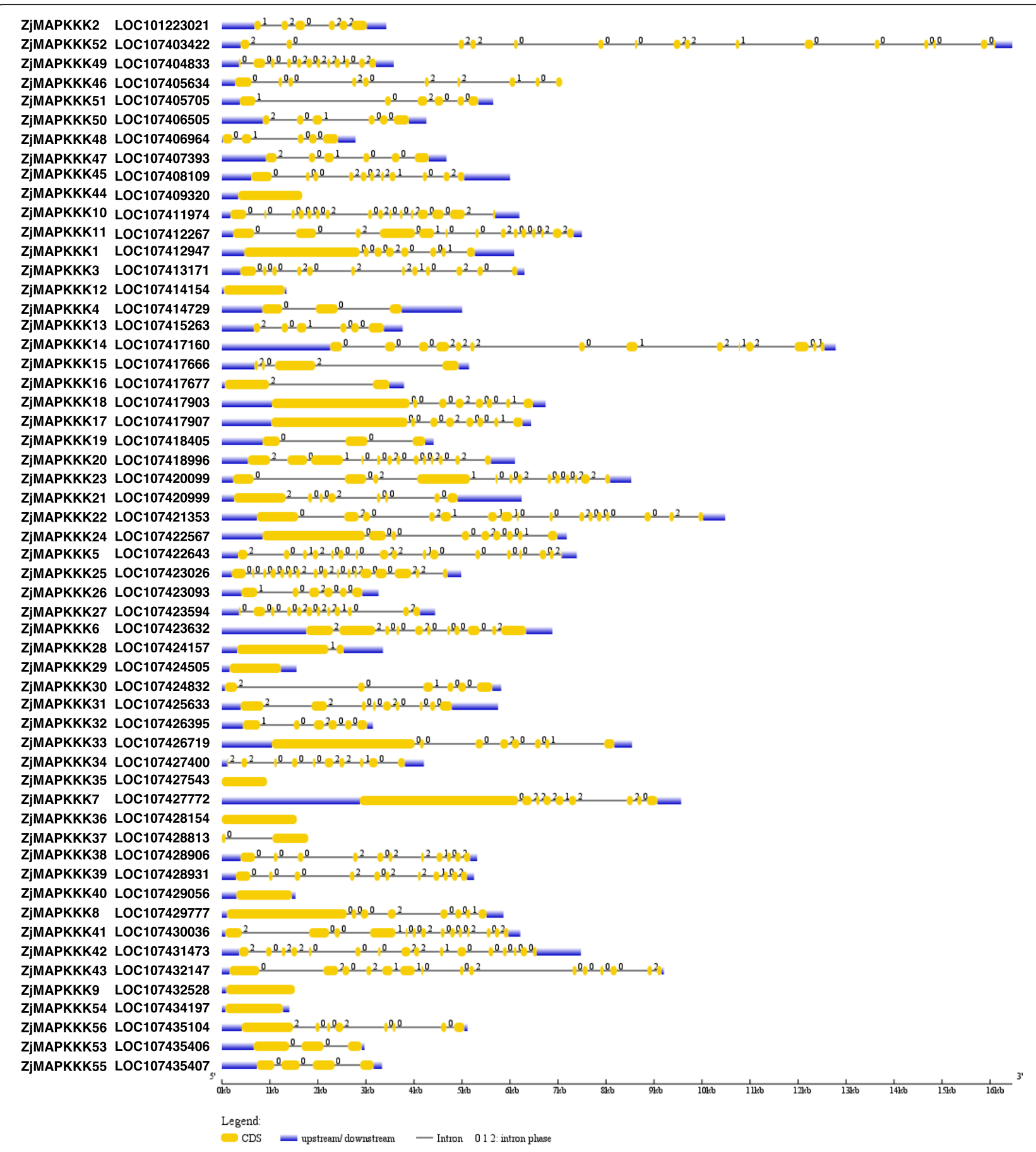

Fig. 3 Schematic diagrams of ZJMAPKKK structures. The yellow and blue boxes and the black lines indicate exons, UTRs and introns, respectively. The numerals 0,1 and 2 indicate different intron phases

in the witches' broom leaves remained approximately constant (Figs. 6, 7 and 8). These results show that ZjMAPKKK26 responds quickly in phyllody leaves and is highly induced in witches' broom leaves, while ZjMAPKKK45 responds more rapidly than
ZjMAPKKK26, as indicated by its high expression in apparently normal leaves in June. In contrast to ZjMAPKKK26 and ZjMAPKKK45, which were significantly upregulated, ZjMAPKKK3, ZjMAPKKK43 and ZjMAPKKK50 were significantly downregulated. 


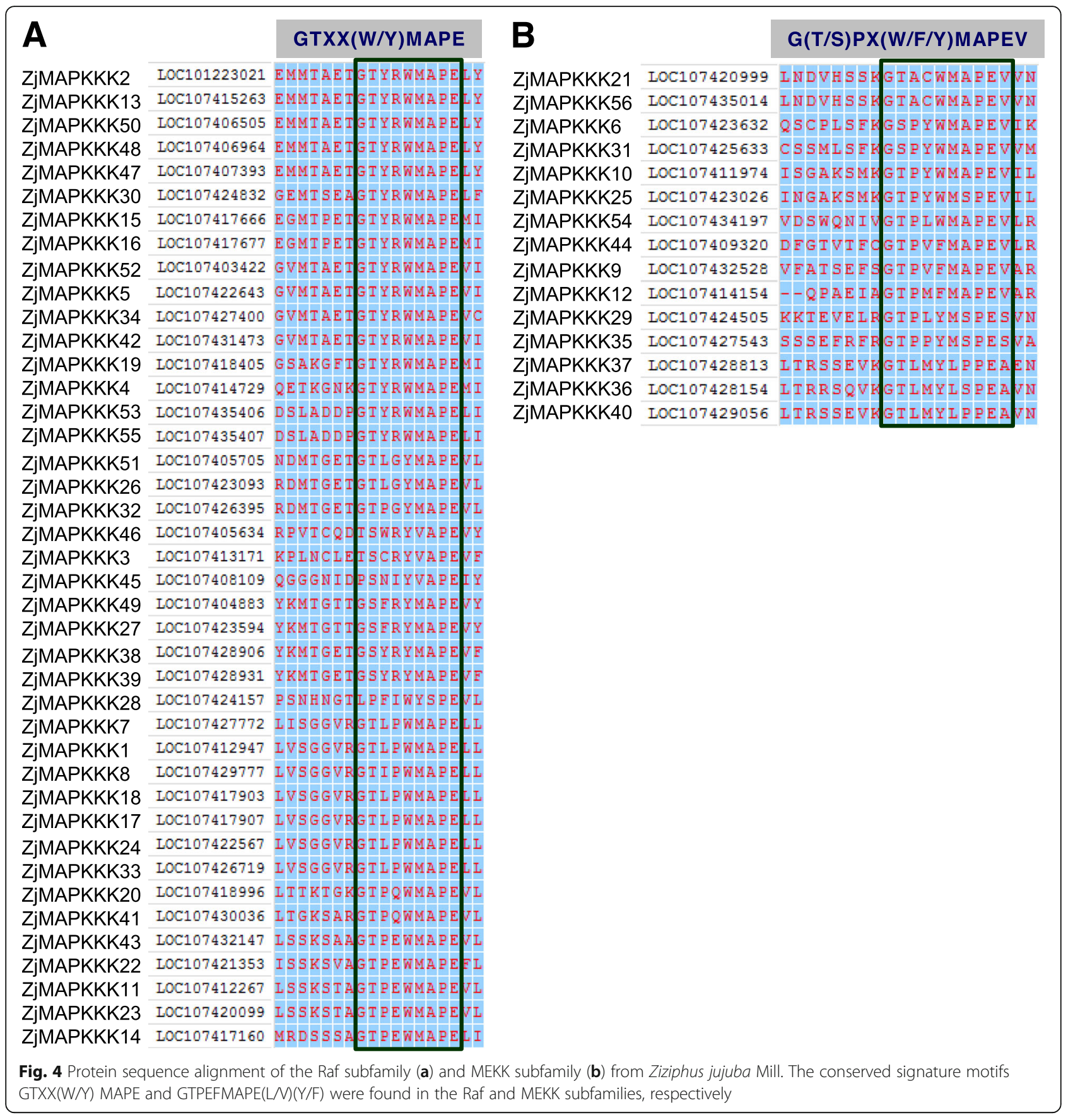

Expression analysis of the ZjMAPKKKs in sterile cultured JWB plantlets

In addition to the ZjMAPKKK expression profiles in the in vivo field tissues, we also examined expression levels in the in vitro cultured JWB plantlets, with uninfected plantlets being used as a control. As shown in Fig. 9, the in vitro ZjMAPKKK expression profiles differed significantly from the in vivo profiles. Only four of the $Z j M A P K K K s$ were significantly induced in the diseased plants - ZjMAPKKK4, - 10, - 25 and - 44. ZjMAPKKK6,
$-7,-17,-18,-30,-34,-35,-37,-40,-41,-43$, $46,-52$ and -53 were significantly downregulated. The other ZjMAPKKKs showed no significant change.

\section{Discussion}

The MAPK cascades are highly conserved, signaltransducing modules found in eukaryotes. These cascades have been widely studied in the plant kingdom, including in Arabidopsis, rice, maize, and apple [5, 9, 12, 13, 15]. Ten MAPKs and five MAPKKs have already been 


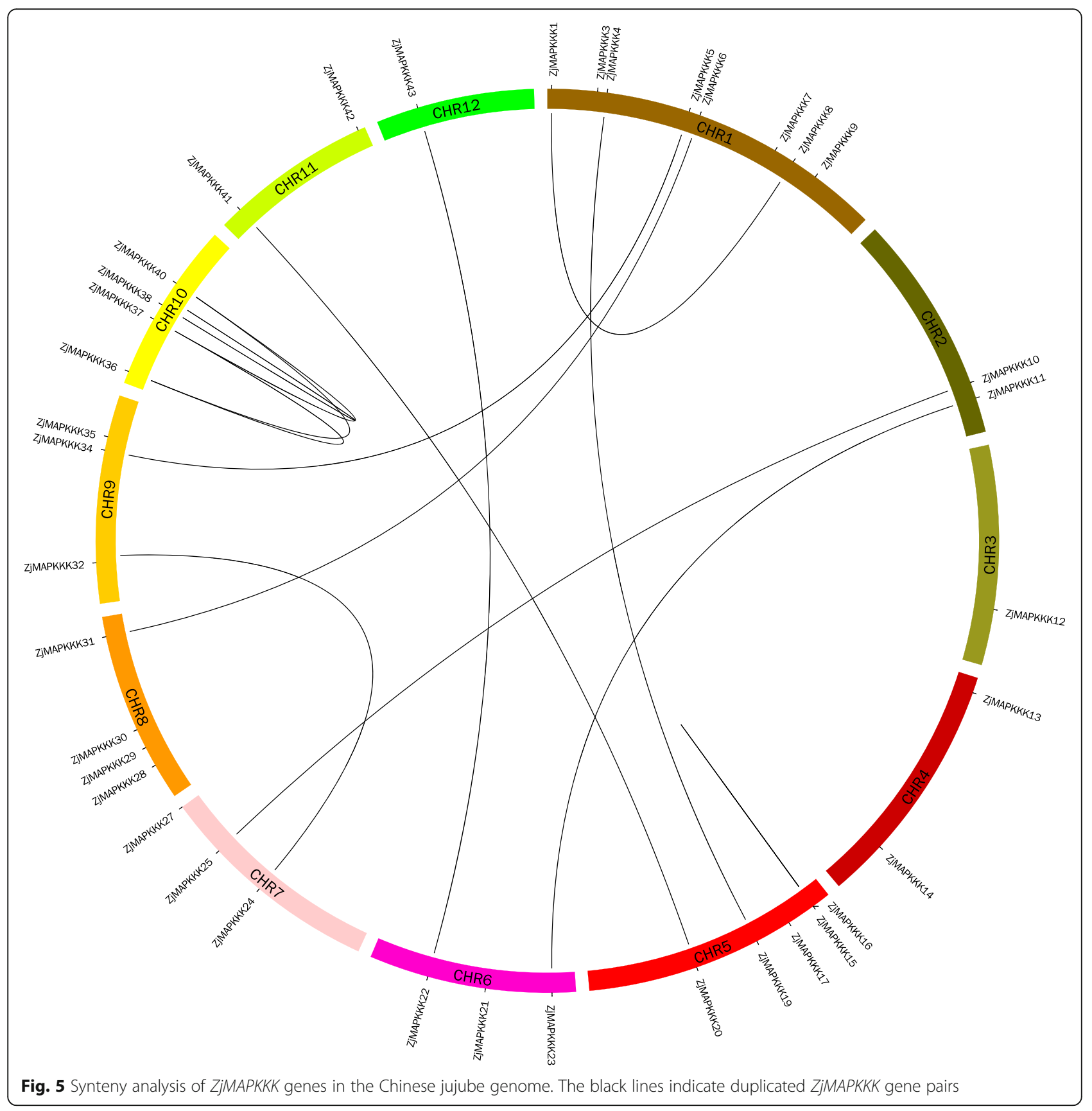

identified in Chinese jujube [17]. The structures of these genes were mostly shown to be similar to those found in other plants (e.g., Arabidopsis, poplar, and apple [17]). In this study, for the first time, we report on MAPKKKs in the Chinese jujube genome. We identified 56 ZjMAPKKKS in Chinese jujube. This number is somewhat larger than that of VviMAPKKKs in grapevine [14] but smaller than that of MdMAPKKKs in apple [15]. The ZjMAPKKKs can be classified into the two main subfamilies Raf and MEKK, with none belonging to the subfamily ZIK. The disappearance of the ZIK subfamily may be due to a loss of function during the evolution of Chinese jujube because the Raf subfamily is considered to be the origin of the MAPKKK family [25]. In addition, the rate of intron loss is known to be more rapid than that of intron gain due to segmental duplication [24]. In this study, we found that the number of conserved motifs and exons was higher in the Raf subfamily than in the MEKK subfamily. We also identified 13 segmental duplication events among 22 ZjMAPKKKs. This finding may indicate that the Raf subfamily contains original genes and that segmental duplication occurred during a long evolutionary history. This interpretation is consistent with that for maize [13]. Furthermore, 43 of the ZjMAPKKKS were widely located over the 12 


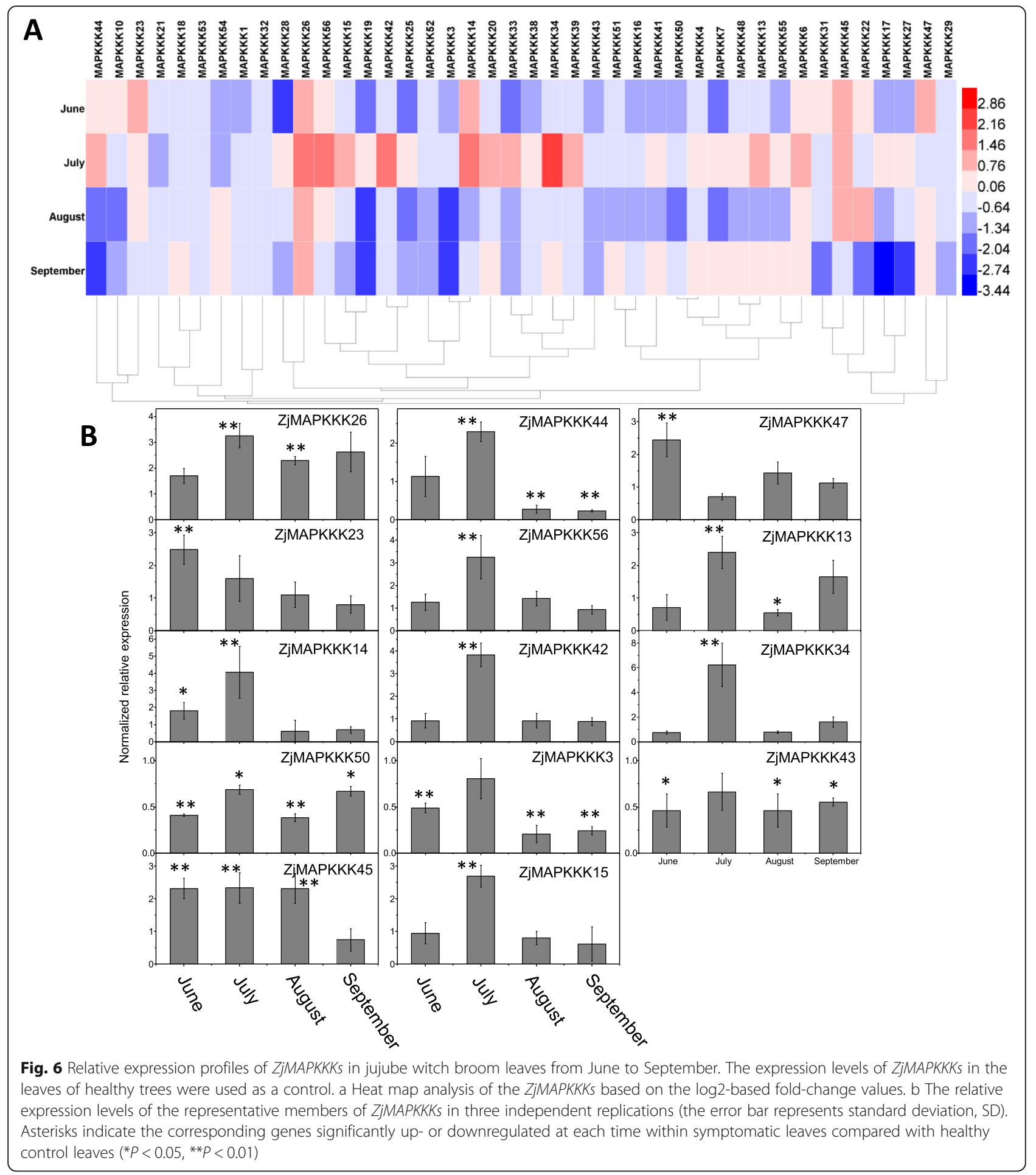

chromosomes, while chromosome positions could not be found for ZjMAPKKK44-56 (Table 1). These findings indicate that evolutionary duplications of ZjMAPKKKs took place and that the unknown locations of many ZjMAPKKKs may confer a number of paralogous genes and play critical roles in various biological processes. For example, ZjMAPKKK44, - 45, - 46, and -50 may be involved in processes associated with phytoplasma infection. This result is different from that obtained in our previous study on ZjMAPKS and ZjMAPKKs, which did not show genome duplication in the evolutionary process [17].

MAPK cascades have been shown to be key signalling modules operating in response to biotic and abiotic stresses, particularly associated with pathogen attack 


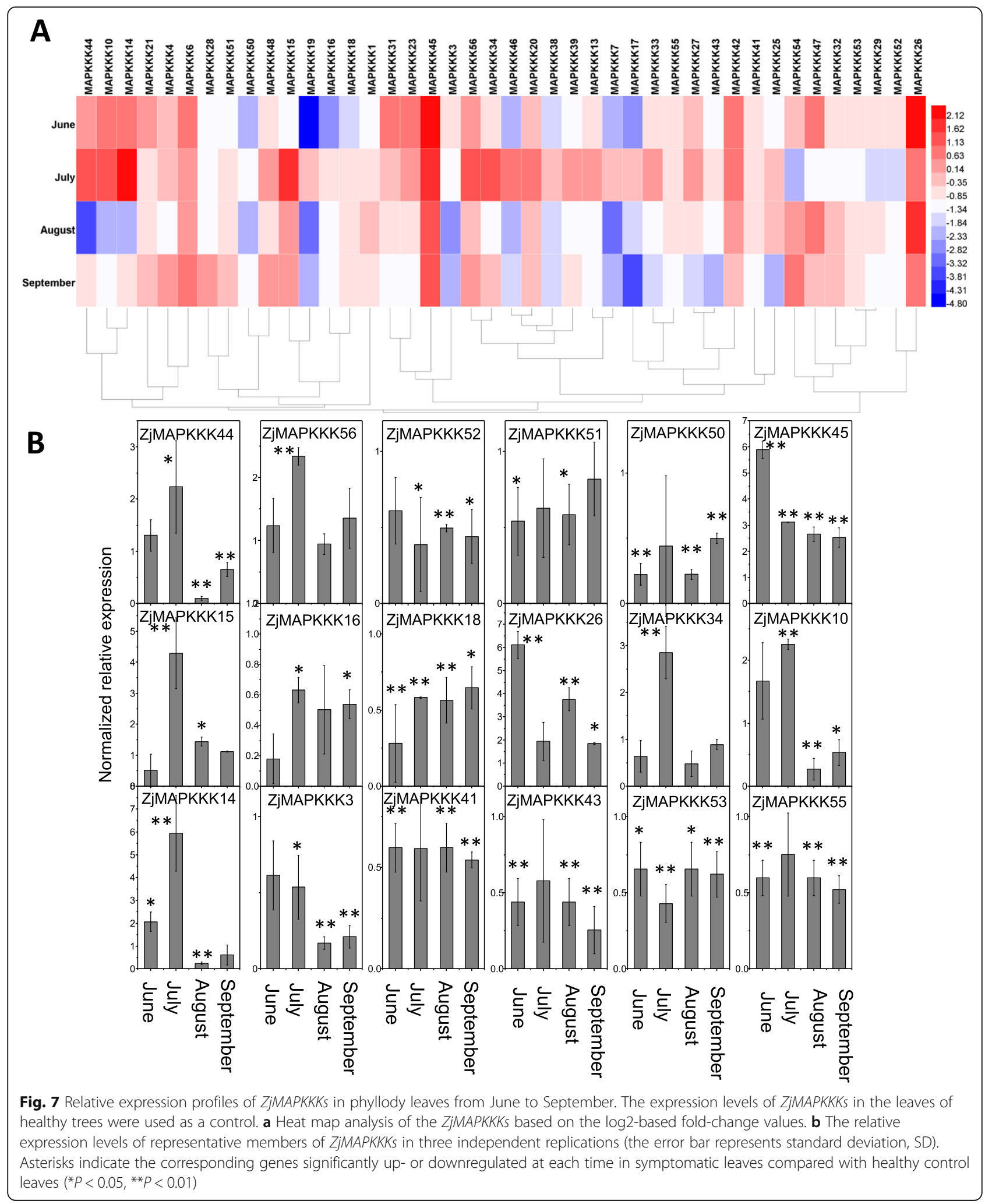

[26]. Among the MAPKKKs, the MEKK subfamily has been widely studied, while the biological functions of the Raf subfamily are still somewhat obscure. In Arabidopsis, the MAPK cascade signalling module MEKK1-MKK4/ $M K K 5-M P K 3 / M P K 6$ is proposed to be activated in the interaction with flg22 treatment [27]. EDR1, a member 


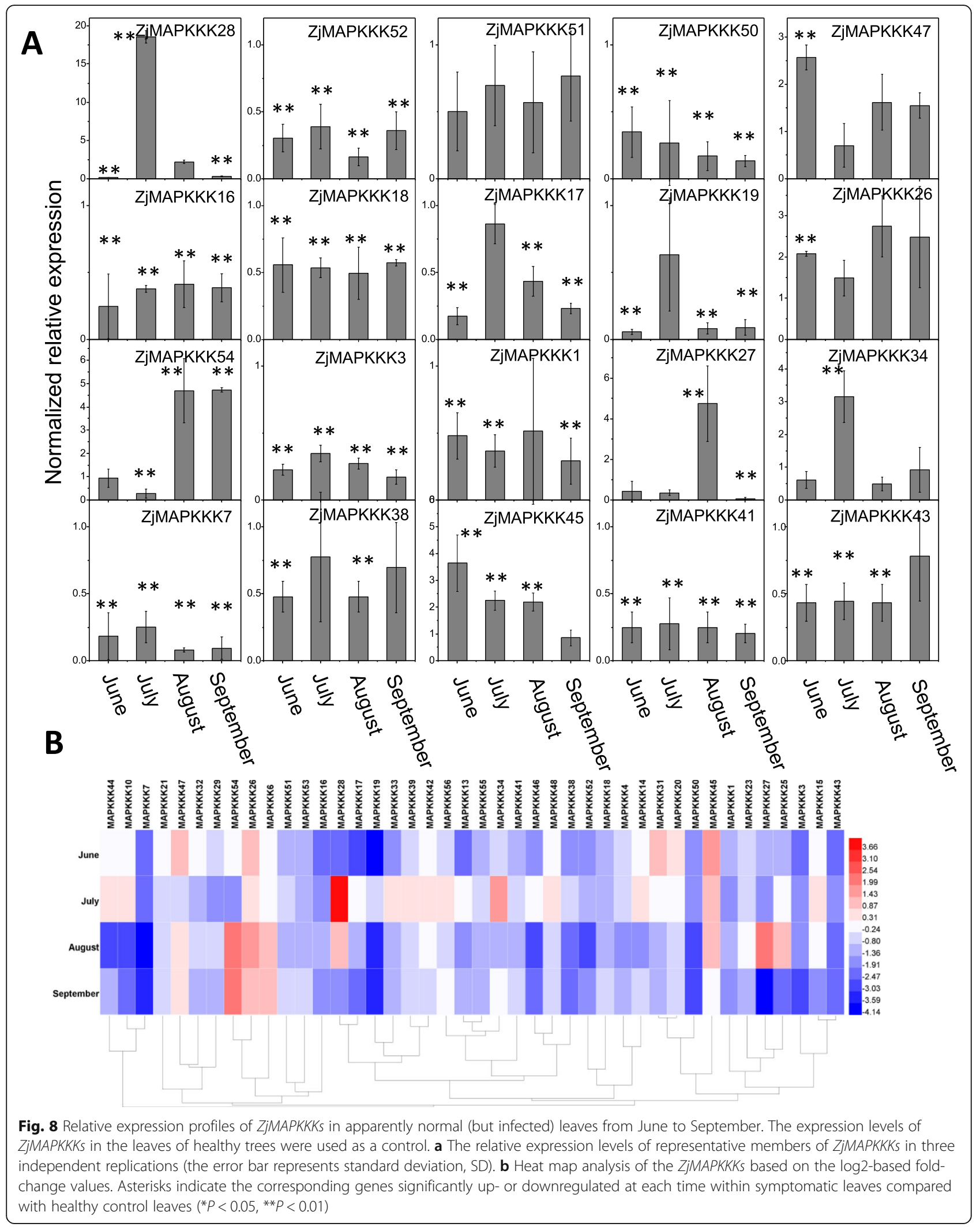




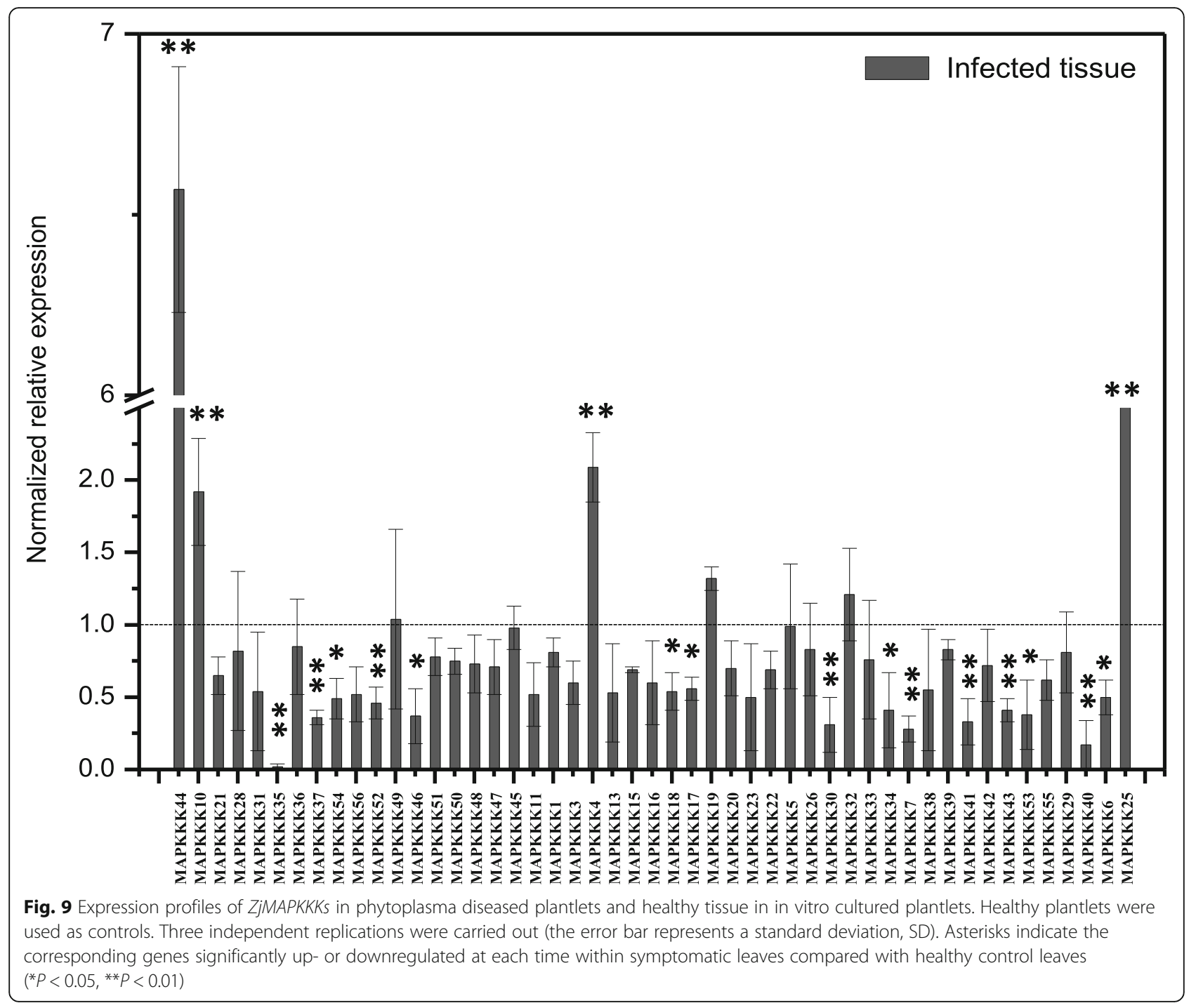

of the Raf subfamily, is responsible for salicylic acidinduced powdery mildew attack [28]. In tomato,

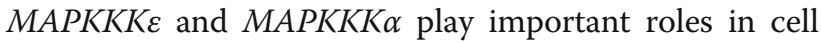
death signalling associated with plant immunity $[29,30]$. In wheat, a MAPKKK named TaFLR can be activated by the leaf rust pathogen Puccinia triticina [31]. In grapevine, VqMAPKKK38 can be highly induced by powdery mildew infection [32]. All the evidence suggests that members of MAPKKKs are essential in pathogen attack signal transduction. In this study, we demonstrate that several ZjMAPKKKs, such as $-26,-45,-3,-43,-50$ etc., might play important role in phytoplasma infection in vivo.. Meanwhile, in the in vitro culture of JWB, four ZjMAPKKKs (ZjMAPKKK4, - 10, -25 and -44) were significantly induced in the diseased plants, while 14 ZjMAPKKKs (ZjMAPKKK6, - 7, - 17, - 18, - 30, - 34, $-35,-37,-40,-41,-43,-46,-52$ and -53$)$ were significantly downregulated. The ZjMAPKKK genes response to phytoplasma were different in vivo and in vitro. This result is consistent with the findings of our previous work in which we found that ZjMPK1 was the gene potentially related to phytoplasma infection in vitro [17] but was different from our later finding that ZjMPK2 was likely regulating along with ZjMEK2 in vivo [23]. The reason behind this phenomenon may be that many more environmental factors (e.g., light and temperature) affect the development of a phytoplasma infection in vivo. Even so, all the candidates discussed above could be involved in phytoplasma infection signal transduction by recruiting the MAPKK and MAPK families. Moreover, ZjMAPKKK43 (homologous to AtRaf1 in Arabidopsis) was downregulated in all the infected tissues, while ZjMAPKKK1O was highly induced in the in vivo JWB plantlets. This gene is homologous to AtMEKK1 in Arabidopsis. Thus, these two genes may both be key to the response to phytoplasma infection in Chinese jujube because AtMEKK1 has already been shown to be important in innate immunity in 
Arabidopsis by activating MKK4/MKK5-MPK3/MPK6 [27]. Moreover, ZjMKK2 could activate ZjMPK2 and thus play essential roles in the JWB defence response [23]. Ye et al. [22] have shown that after phytoplasma infection, MAPKs can also be activated and that the transcription factor WRKY33 is regulated. Taken together, these results indicate the likely $Z j M A P K K K s$ that mediate $Z j M K K 2-Z j M P K 2$ to $W R K Y$ transcription factors in response to phytoplasma infection, which will need to be resolved in future studies. In addition, the scaffold $R A C K$ 1 (Receptor for Activated C Kinase 1) has been identified in Arabidopsis, which tethers MAPKKKs to the plasma membrane and associates with the Gb subunit involved in immune responses [33]. Therefore, in future studies, it may be worth investigating the functions of these $M A P K K K$ candidates and their relationships to RACK1 in response to phytoplasma infection of Chinese jujube.

\section{Conclusions}

Using a range of informatics analyses of MAPKKKs in the Chinese jujube genome, 56 members were identified and named ZjMAPKKK according to their locations on the chromosomes. The phylogeny, conserved motifs and intron/exon analyses confirm the identity of these kinases as members of each subfamily. The expression profiles of the ZjMAPKKKs were recorded by qPCR in materials at four timings and exhibiting three levels of JWB symptoms (witches' broom leaves, phyllody leaves and apparently normal leaves) and in sterile in vitro cultures of JWB plantlets. ZjMAPKKK26 and - 45 were significantly upregulated and ZjMAPKKK $3,-43$ and -50 were downregulated in the three main infected tissues. Meanwhile, in the sterile cultivated tissues of JWB plantlets, four ZjMAPKKKs (4, 10, 25 and -44$)$ were significantly induced in the diseased plants. Additionally, ZjMAPKKK6, - 7, - 17, - 18, - 30, - 34, - 35, $-37,-40,-41,-43,-46,-52$ and -53 were significantly downregulated. Our results provide early insight into certain ZjMAPKKKs that could be involved in the plant response to phytoplasma infection.

\section{Methods}

\section{Identification of ZjMAPKKKs in Chinese jujube}

The ZjMAPKKKS gene family was identified according to our previous study on the identification of ZjMAPKKs and $Z j M A P K s$ with some modifications [17]. First, the whole protein sequences of MAPKKKs in Arabidopsis were retrieved from TAIR databases (Additional file 1 Figure S1). These sequences were used as queries to search against the whole jujube genome database (accession JREP00000000) [34]. In addition, the alignments of all Arabidopsis MAPKKK sequences were used to construct an HMM profile (http://hmmer.org/download.html) to search for other potential MAPKKK members in the jujube genome database. All the potential ZjMAPKKK genes that contain a protein kinase domain (PF00069) were confirmed by HMMER tools [35], redundancy was removed, and the remaining sequences were identified as belonging to the ZjMAPKKK family. Furthermore, the ExPASy Proteomics Server (http://expasy.org/) was used to calculate the theoretical pI (isoelectric point) and $\mathrm{Mw}$ (molecular weight) of the putative ZjMAPKKKs [36].

\section{Gene structure, protein domain and motif analyses of ZjMAPKKK genes}

The open reading frames (ORFs) of 56 ZjMAPKKKS were analysed through the National Center for Biotechnology Information (NCBI, http://www.ncbi.nlm.nih.gov) ORF finder, and the main protein domains of ZjMAPKKKs were also recorded by blastp of NCBI (Additional file 2Table S1). GSDS (Gene Structure Display Server, http://gsds.cbi.pku.edu.cn/) was used to determine the exon/intron structures of individual ZjMAPKKKs by aligning the cDNA sequences with their corresponding genomic DNA sequences [37]. The MEME database was used to identify the conserved motifs of ZjMAPKKKS [38]. The ideal motif widths were set to between 6 and 50, and each protein sequence of the $Z j M A P K K K s$ subfamily was used to determine the highest number of conserved domains [39].

\section{Sequence alignment, phylogenetic and gene duplication analysis}

All the protein sequences of ZjMAPKKKs were aligned by ClusterX software using the default parameter values. The alignments of the protein sequences of 56 ZjMAPKKKs and 80 AtMAPKKKs were then subjected to phylogenetic analysis using MEGA 6.06 alignment explorer. The parameters of alignment were as follows: gap opening penalty, 10.00; gap extension penalty, 0.50 (both in pairwise alignment and in multiple alignment); protein weight matrix, gonnet; residue-specific penalties, on; hydrophilic penalties, on; gap separation distance, 0 ; end-gap separation, on; use negative matrix, off; and delay divergent cutoff (\%), 30. Phylogenetic trees were constructed by the neighbour-joining (NJ) method. The parameters of the constructed trees were as follows: statistical method, neighbour-joining; scope, all selected taxa; test of phylogeny, bootstrap method; number of bootstrap replications, 1000; substitution types, amino acid; model/method, Poisson model; rates among sites, uniform rates; pattern among lineages, same (homogeneous); and gaps/missing data treatment: complete deletion. The Multiple Collinearity Scan toolkit (MCScanX) was used to analyse the gene duplication events, with the default parameters and the homologous relationships being drawn using Circos software [40, 41]. 
Plant materials and treatments

Ziziphus jujuba Mill. 'Dongzao' was cultivated in the Experimental Station of Chinese Jujube, Hebei Agricultural University and used for experimental material. For each time point, leaf samples were collected from at least three healthy jujube trees and from three trees infected with JWB. All experimental trees were cultivated under natural environmental conditions [19, 21]. Each year, visual symptoms of JWB, such as phyllody leaves (floral organs becoming leaf-like, middle severity) and apparently normal leaves (infected but asymptomatic, or exhibiting minimal symptoms), were first observed in early June. Later, witches' broom leaves (shoots with small leaves, maximum severity) were observed in the middle of June. Finally, mature witches' broom leaves, phyllody leaves and apparently normal leaves were collected from the same branch of diseased trees in June, July, August, and September (on the 15th day of each month). The leaves from healthy trees were used as controls for each time point. The use of the above time course is that the phytoplasma content of the branches increased dramatically during the period, reaching a peak in July and August and declining thereafter [19]. The different types of materials are shown in Additional file 3 Figure S2. Detection of JWB phytoplasma in the infected materials employed DAPI staining at the histological level and quantitative real-time PCR analysis (qRT-PCR) at the molecular level [21]. Each material was sampled with three replicates, one from each of three different trees. In addition, sterile cultivated JWB diseased plantlets of Ziziphus jujuba Mill. 'Goutouzao' were used as a parallel testing group, with healthy plantlets being used as controls (as shown in Additional file 4 Figure S3). The presence of JWB phytoplasma in the diseased and healthy sterile cultivated plantlets was detected using DAPI staining at the histological level (Additional file 5 Figure S4) [23]. Ten plantlets were pooled to form one sample, and three independent biological replications were sampled separately. All samples were frozen rapidly in liquid nitrogen and held at $-80^{\circ} \mathrm{C}$ pending RNA isolation and qPCR analyses.

\section{RNA extraction and qRT-PCR analyses}

Total RNA was extracted from the leaves using the TIANGEN RNA Extraction Kit. Genomic DNA contamination was removed by digestion with DNase. cDNA synthesis was carried out with the TaKaRa RNA PCR Kit (AMV) Ver. 3.0 (TaKaRa) according to the manufacturer's protocol using $1 \mu \mathrm{g}$ of RNA template.

qRT-PCR was carried out on the Bio-Rad $\mathrm{iQ}^{\mathrm{m}} 5$ using TransStart Top Green qPCR SuperMix AQ131 (TransGen Biotech, China). The $20-\mu \mathrm{L}$ reaction system contained $10 \mu \mathrm{L}$ of $2 \times$ SYBR Premix ExTaq ${ }^{\mathrm{Tm}}, 0.4 \mu \mathrm{L}$ each of $10 \mu \mathrm{M}$ primers, $1 \mu \mathrm{L}$ diluted cDNA and $8.2 \mu \mathrm{L} \mathrm{ddH}_{2} \mathrm{O}$.
The thermal profile was preincubated for $3 \mathrm{~min}$ at $94{ }^{\circ} \mathrm{C}$ followed by 40 cycles of $5 \mathrm{~s}$ at $94{ }^{\circ} \mathrm{C}, 15 \mathrm{~s}$ at $55 \sim 63^{\circ} \mathrm{C}$ and $15 \mathrm{~s}$ at $72^{\circ} \mathrm{C}$. Relative expression levels of $Z j M A P K K K s$ were calculated by the $2^{-\Delta \Delta \mathrm{Ct}}$ method [40] using ZjActin as an endogenous control for normalisation [41]. The primer sequences of ZjMAPKKKs for qPCR are shown in Additional file 6 Table S2.

\section{Heatmap construction}

The expression profiles of all ZjMAPKKKs in the different samples are illustrated by a colour gradient heatmap. The heatmap was constructed by heatmap software Heml 1.0 using log2-based expression fold changes.

\section{Statistical analyses}

All data were analysed by the two-sample t-test method using Origin 8.0 software with range tests $(P<0.05$ and $P<0.01)$.

\section{Supplementary information}

Supplementary information accompanies this paper at https://doi.org/10. 1186/s12864-020-6548-6. Additional file 1: Figure S1. Protein sequences of MAPKKKs from
Ziziphus jujuba Mill. and Arabidopsis thaliana.

Additional file 2: Table S1. Number of main protein domains of ZjMAPKKKs.

Additional file 3: Figure S2. Healthy and diseased in vitro plantlets. A: Healthy plantlets; B: Diseased plantlets.

Additional file 4: Figure S3. Tissues showing different JWB disease symptoms. A: Witches' broom leaves; B: Phyllody leaves; C: Apparently normal leaves; D: Healthy leaves. A, B and C were used as a test group collected from diseased trees. D was used as a control collected from healthy trees.

Additional file 5: Figure S4. Determination of phytoplasma in the sieve element in jujube petiole phloem by using 4',6-diamidino-2phenylindole (DAPI). A, No fluorescent spots were observed in the sieve element (SE) of healthy plantlets. B, The fluorescent spots formed a large, bright circle in the sieve element (SE) of the diseased plantlets. The numbers and sizes of the fluorescent spots indicate the number of phytoplasmas. Bar $=100 \mu \mathrm{m}$.

Additional file 6: Table S2. Primer sequences of ZjMAPKKKKs for qRTPCR

Additional file 7: Figure S5. CDS sequences of ZjMAPKKKS

Additional file 8: Table S3. Number of introns and exons of ZjMAPKKK genes

Additional file 9: Table S4. Fold-change values of ZjMAPKKKS in the leaves of witches' broom, phyllody and apparently normal symptoms.

\section{Abbreviations}

Chr: Chromosome.; JWB: Jujube witches' broom; MAPK: Mitogen-activated protein kinase; MAPKK: MAPK kinase; MAPKKK: MAPK kinase kinase; MW: The theoretical molecular weight of proteins.; ORF: Open reading frames.; PI: The theoretical isoelectric point of proteins.; qPCR: Quantitative real-time PCR.; RACK 1: Receptor for Activated C Kinase 1.; S-TKc: Serine/threonine protein kinase; Zj: Ziziphus jujuba.

Acknowledgements

Not applicable. 


\section{Ethical standards}

This research does not contain any studies with human participants or animals.

\section{Authors' contributions}

$\mathrm{JZ}$ and $\mathrm{ML}$ designed the research and wrote the paper. ZL and LW performed the experiments, analyzed the data and wrote the paper. $C X, Y C$, WG and YZ performed the experiments and participated in the data analysis. All authors read and approved the final the manuscript.

\section{Funding}

This work was supported by the Funds for Hebei Distinguished Young Scholar (C2016204145), the Youth Fund of Hebei Province Natural Science Foundation (C2016204157), Green Channel Fund of Hebei Province Natural Science Foundation (C2019204308), the Significance Fund of Hebei Province Natural Science Foundation (C2017204114), and the Agricultural University of Hebei Foundation for Leaders of Disciplines in Science Technology, Funds for Young Talent Project of Hebei Agricultural University Foundation (grant number YJ201853). Funds were used for the design of the study and collection, analysis, and interpretation of data and in writing the manuscript, as well as in the open access payment.

\section{Availability of data and materials}

All data and materials are presented in the main paper and additional file. In addition, the whole protein sequences of MAPKKKs in Arabidopsis were retrieved from TAIR databases. The CDS and genome sequences of MAPKKKS in jujube were retrieved from the whole jujube genome database (accession JREP00000000) in NCBI.

\section{Ethics approval and consent to participate}

The healthy and diseased jujube trees used in this study were from the Experimental Station of Chinese Jujube, Hebei Agricultural University, in Baoding, Hebei. Chinese jujube is one of traditional and widespread fruit trees in China, and it is not an endangered species. No specific permits are required for sample collection on Chinese jujube.

\section{Consent for publication}

Not applicable.

\section{Competing interests}

The authors declare that they have no competing interests.

\section{Author details}

'College of Horticulture, Hebei Agricultural University, Baoding, China. ${ }^{2}$ Research Center of Chinese Jujube, Hebei Agricultural University, Baoding, China. ${ }^{3}$ College of Life Science, Hebei Agricultural University, Baoding, China. ${ }^{4}$ Key Laboratory of Hebei Province for Plant Physiology and Molecular Pathology, Hebei Agricultural University, Baoding, Hebei, China.

\section{Received: 15 May 2019 Accepted: 31 January 2020}

Published online: 10 February 2020

\section{References}

1. Rodriguez MCS, Petersen M, Mundy J. Mitogen-activated protein kinase signaling in plants. Annu Rev Plant Biol. 2010;61:621-49.

2. Sinha AK, Jaggi M, Raghuram B, Tuteja N. Mitogen-activated protein kinase signaling in plants under abiotic stress. Plant Signal Behav. 2011;6(2):196203.

3. Galletti R, Ferrari S, De Lorenzo G. Arabidopsis MPK3 and MPK6 play different roles in basal and Oligogalacturonide- or Flagellin-induced resistance against Botrytis cinerea. Plant Physiol. 2011;157(2):804-14.

4. Jonak C, Okresz L, Bogre L, Hirt H. Complexity, cross talk and integration of plant MAP kinase signalling. Curr Opin Plant Biol. 2002;5(5):415-24.

5. Group M. Mitogen-activated protein kinase cascades in plants: a new nomenclature. Trends Plant Sci. 2002;7(7):301-8.

6. Zhang S, Klessig DF. MAPK cascades in plant defense signaling. Trends Plant Sci. 2001;6(11):520-7.

7. Asai T, Tena G, Plotnikova J, Willmann MR, Chiu WL, Gomez-Gomez L, Boller T, Ausubel FM, Sheen J. MAP kinase signalling cascade in Arabidopsis innate immunity. Nature. 2002;415(6875):977-83.
8. Pitzschke A, Djamei A, Bitton F, Hirt H. A major role of the MEKK1-MKK1/2MPK4 pathway in ROS signalling. Mol Plant. 2009;2(1):120-37.

9. $\quad$ Kong Q, Qu N, Gao MH, Zhang ZB, Ding XJ, Yang F, Li YZ, Dong OX, Chen $\mathrm{S}$, Li X. The MEKK1-MKK1/MKK2-MPK4 kinase Cascade negatively regulates immunity mediated by a mitogen-activated protein kinase kinase kinase in Arabidopsis. Plant Cell. 2012;24(5):2225-36.

10. Liu Y, Schiff M, Dinesh-Kumar SP. Involvement of MEK1 MAPKK, NTF6 MAPK, WRKY/MYB transcription factors, COI1 and CTR1 in N-mediated resistance to tobacco mosaic virus. The Plant J. 2004;38(5):800-9.

11. Ekengren SK, Liu Y, Schiff M, Dinesh-Kumar SP, Martin GB. Two MAPK cascades, NPR1, and TGA transcription factors play a role in Pto-mediated disease resistance in tomato. The Plant J. 2003;36(6):905-17.

12. Rao KP, Richa T, Kumar K, Raghuram B, Sinha AK. In silico analysis reveals 75 members of mitogen-activated protein kinase kinase kinase gene family in rice. DNA Res. 2010;17(3):139-53.

13. Liu Y, Zhou M, Gao Z, Ren W, Yang F, He H, Zhao J. RNA-Seq analysis reveals MAPKKK family members related to drought tolerance in maize. PLoS One. 2015;10(11):e0143128.

14. Wang G, Lovato A, Polverari A, Wang M, Liang YH, Ma YC, Cheng ZM. Genomewide identification and analysis of mitogen activated protein kinase kinase kinase gene family in grapevine (Vitis vinifera). BMC Plant Biol. 2014;14:219.

15. Sun M, Xu Y, Huang J, Jiang Z, Shu H, Wang H, Zhang S. Global identification, classification, and expression analysis of MAPKKK genes: functional characterization of MdRaf5 reveals evolution and droughtresponsive profile in apple. Sci Rep. 2017;7(1):13511.

16. Wang $L$, Hu W, Tie W, Ding Z, Ding X, Liu Y, Yan Y, Wu C, Peng M, Xu B. The MAPKKK and MAPKK gene families in banana: identification, phylogeny and expression during development, ripening and abiotic stress. Sci Rep. 2017; $7(1): 1159$

17. Liu ZG, Zhang LM, Xue CL, Fang H, Zhao J, Liu MJ. Genome-wide identification and analysis of MAPK and MAPKK gene family in Chinese jujube (Ziziphus jujuba mill.). BMC Genomics. 2017;18.

18. Jung HY, Sawayanagi T, Kakizawa S, Nishigawa H, Wei W, Oshima K, Miyata S, Ugaki M, Hibi T, Namba S. Candidatus Phytoplasma ziziphi', a novel phytoplasma taxon associated with jujube witches'-broom disease. Int J Syst Evol Micr. 2003:53:1037-41.

19. Liu MJ, Zhao J, Zhou JY. Jujube Witches' broom disease. China Agriculture Press, Beijing, 2010 (In Chinese).

20. Lee S, Kim CE, Cha B. Migration and distribution of graft-inoculated jujube Witches'-broom Phytoplasma within a Cantharanthus roseus plant. Plant Pathology J. 2012;28(2):191-6.

21. Xue C, Liu Z, Dai L, Bu J, Liu M, Zhao Z, Jiang Z, Gao W, Zhao J. Changing host photosynthetic, carbohydrate, and energy metabolisms play important roles in Phytoplasma infection. Phytopathology. 2018;108(9):1067-77.

22. Ye X, Wang HY, Chen P, Fu B, Zhang MY, Li JD, Zheng XB, Tan B, Feng JC. Combination of iTRAQ proteomics and RNA-seq transcriptomics reveals multiple levels of regulation in phytoplasma-infected Ziziphus jujuba mill. Hortic Res-England. 2017;4.

23. Liu ZG, Zhao Z, Xue CL, Wang LL, Wang LL, Feng C, Liu MJ. Three Main genes in the MAPK Cascade involved in the Chinese jujube-Phytoplasma interaction. Forests. 2019;10(5):392.

24. Nuruzzaman M, Manimekalai R, Sharoni AM, Satoh K, Kondoh H, Ooka H, Kikuchi S. Genome-wide analysis of NAC transcription factor family in rice. Gene. 2010;465(1-2):30-44.

25. Ye JQ, Yang $H$, Shi HT, Wei YX, Tie WW, Ding ZH, Yan Y, Luo Y, Xia ZQ, Wang WQ. The MAPKKK gene family in cassava: genome-wide identification and expression analysis against drought stress. Sci Rep. 2017;7.

26. Pitzschke A, Schikora A, Hirt H. MAPK cascade signalling networks in plant defence. Curr Opin Plant Biol. 2009;12(4):421-6.

27. Colcombet J, Hirt H. Arabidopsis MAPKs: a complex signalling network involved in multiple biological processes. Biochem J. 2008;413:217-26.

28. Wu GH, Liu SM, Zhao YF, Wang W, Kong ZS, Tang DZ. ENHANCED DISEASE RESISTANCE4 associates with CLATHRIN HEAVY CHAIN2 and modulates plant immunity by regulating relocation of EDR1 in Arabidopsis. Plant Cell. 2015;27(3):857-73.

29. del Pozo O, Pedley KF, Martin GB. MAPKKK alpha is a positive regulator of cell death associated with both plant immunity and disease. EMBO J. 2004; 23(15):3072-82.

30. Melech-Bonfil S, Sessa G. Tomato MAPKKK epsilon is a positive regulator of cell-death signaling networks associated with plant immunity. Plant J. 2010; 64(3):379-91. 
31. Gao Y, Stebbing J, Tubei K, Tian LN, Li XQ, Xing T. Response of TaFLR MAPKKK to wheat leaf rust and Fusarium head blight and the activation of downstream components. Trop Plant Pathol. 2016;41(1):15-23.

32. Jiao YT, Wang D, Wang L, Jiang CY, Wang YJ. VqMAPKKK38 is essential for stilbene accumulation in grapevine. Hortic Res-England. 2017;4.

33. Su JB, Xu J, Zhang SQ. RACK1, scaffolding a heterotrimeric $\mathrm{G}$ protein and a MAPK cascade. Trends Plant Sci. 2015;20(7):405-7.

34. Liu MJ, Zhao J, Cai QL, Liu GC, Wang JR, Zhao ZH, Liu P, Dai L, Yan GJ, Wang WJ. The complex jujube genome provides insights into fruit tree biology. Nat Commun. 2014;5.

35. Wang J, Pan CT, Wang Y, Ye L, Wu J, Chen LF, Zou T, Lu G. Genome-wide identification of MAPK, MAPKK, and MAPKKK gene families and transcriptional profiling analysis during development and stress response in cucumber. BMC Genomics. 2015;16.

36. Gasteiger E, Gattiker A, Hoogland C, Ivanyi I, Appel RD, Bairoch A. Expasy: the proteomics server for in-depth protein knowledge and analysis. Nucleic Acids Res. 2003:31(13):3784-8.

37. Guo AY, Zhu QH, Chen X, Luo JC. [GSDS: a gene structure display server]. Yi chuan $=$. Hereditas. 2007;29(8):1023-6.

38. Bailey TL, Elkan C. Fitting a mixture model by expectation maximization to discover motifs in biopolymers. Proc Int Conf Intell Syst Mol Biol. 1994;2:28-36.

39. Neupane A, Nepal MP, Piya S, Subramanian S, Rohila JS, Reese RN, Benson BV. Identification, nomenclature, and evolutionary relationships of mitogenactivated protein kinase (MAPK) genes in soybean. Evol Bioinformatics Online. 2013;9:363-86.

40. Livak KJ, Schmittgen TD. Analysis of relative gene expression data using real-time quantitative PCR and the 2(T)(-Delta Delta C) method. Methods. 2001;25(4):402-8

41. Bu J, Zhao J, Liu M. Expression Stabilities of Candidate Reference Genes for RT-qPCR in Chinese Jujube (Ziziphus jujuba Mill.) under a Variety of Conditions. Plos One. 2016;11(4):e0154212

\section{Publisher's Note}

Springer Nature remains neutral with regard to jurisdictional claims in published maps and institutional affiliations.

Ready to submit your research? Choose BMC and benefit from:

- fast, convenient online submission

- thorough peer review by experienced researchers in your field

- rapid publication on acceptance

- support for research data, including large and complex data types

- gold Open Access which fosters wider collaboration and increased citations

- maximum visibility for your research: over $100 \mathrm{M}$ website views per year

At $\mathrm{BMC}$, research is always in progress.

Learn more biomedcentral.com/submissions 